\title{
Unexpected Tonsillar Carcinoma in a Sleep Apnea Patient - Is Routine Histopathology After Tonsillectomy Avoidable?
}

\author{
Jan Plzák ${ }^{*}, 1,2,3$, Michal Zábrodský ${ }^{1}$, Jan Kastner ${ }^{1}$, Jan Klozar ${ }^{1}$ and Jan Betka ${ }^{1}$ \\ ${ }^{I}$ Department of Otorhinolaryngology and Head and Neck Surgery, $1^{\text {st }}$ Faculty of Medicine, Charles University, Faculty \\ Hospital Motol, Prague, Czech Republic \\ ${ }^{2}$ Institute of Anatomy, $I^{\text {st }}$ Faculty of Medicine, Charles University, Prague, Czech Republic \\ ${ }^{3}$ Center of Cell Therapy and Tissue Repair, $2^{\text {nd }}$ Faculty of Medicine, Charles University, Prague, Czech Republic
}

\begin{abstract}
Tonsillectomy specimens are routinely sent for histopathologic evaluation to assess inflammatory process as well as to exclude occult malignancy. But nowadays many authors believe that routine microscopic examination of tonsillectomy specimen results in unnecessary cost and consumption of resources and time because of a low incidence of malignant tumor in unsuspected tonsils. In sleep surgery "healthy" tonsils are operated. We report on a case in which tonsillar carcinoma was detected after tonsillectomy for obstructive sleep apnea. The patient fortunately did not require any adjuvant therapy. A presented case of occult malignancy of normal size tonsil in the sleep apnea patient without any risk factors together with recent literature review support our recommendation: The routine histopathologic evaluation of surgically removed tonsillar tissue in the absence of preoperative risk factors is not necessary.
\end{abstract}

Keywords: Obstructive sleep apnea, surgery, tonsillectomy, histopathology, occult malignancy, carcinoma of the tonsil.

\section{INTRODUCTION}

Obstructive sleep apnea is a common indication of tonsillectomy. In sleep surgery "healthy" tonsils are operated. Tonsillectomy specimens are routinely sent for histopathologic evaluation to assess inflammatory process as well as to exclude subclinical malignancy. Since an effort is focused on reduction of healthcare costs, necessity for this routine examination is currently frequently judged. We report on a case in which tonsillar carcinoma was detected after tonsillectomy for sleep apnea.

\section{CASE}

A man, 53 years old, nonobese (body-mass index 25.1), nonsmoker, without alcohol abuse, suffering from moderate obstructive sleep apnea with apnea-hypopnea index (AHI) 29.5 refused CPAP (continuous positive airway pressure). Therefore he was recommended for surgical treatment. He had a symmetric hypertrophy of the tonsils, covered by normal mucosa, palatal webbing, prolonged uvula, and septal deviation. There was a narrowing of the retrolingual space confirmed by cephalometry, posterior airway space $=7 \mathrm{~mm}$. No cervical lymphadenopathy. Uvulopalatopharyngoplasty (UPPP) with uvulopalatal flap [1] plus bilateral tonsillectomy, radiofrequency surgery of the base of the tongue, and septoplasty under general anesthesia were performed. Perioperative and immediate postoperative course passed without extraordinary event. The patient complained of adequate odynophagia after UPPP, with no

*Address correspondence to this author at the Department of Otorhinolaryngology and Head and Neck Surgery, $1^{\text {st }}$ Faculty of Medicine, Charles University, Faculty Hospital Motol, V Úvalu 84, 15006 Prague 5, Czech Republic; Tel: +420 224434301; Fax: +420 224434319;

E-mail: jan.plzak@lf1.cuni.cz side domination. However, microscopic examination was surprising. It revealed a subepithelially located nest of mucoepidermoid carcinoma in the left tonsillectomy specimen $(3.5 \times 4 \mathrm{~mm})$, surrounded by an intact tonsillar tissue (stage T1N0). Clinical examination two weeks after the surgery showed an asymmetry of the faucial isthmus with smooth dishing on the left side. MRI T2W described suspicious tumorous infiltration of the left pharyngeal wall $(22 \times 15 \times 22 \mathrm{~mm})$ with enlargement of the regional neck lymph nodes $(15 \mathrm{~mm})$ in the level II (Fig. 1). Biopsy from the suspected pharyngeal region under local and later under general anesthesia certified no evidence of malignant cells. Careful follow-up was performed. Three months after the surgery there was no asymmetry of the pharynx based on both clinical examination and MRI (Fig. 2). Sleep study six month after the treatment showed significant reduction of AHI (AHI 8.8). Using Sher's criteria [2] for obstructive sleep apnea (final AHI $<20$ and AHI reduction $>50 \%$ ), the patient was classified as cured. After five years the patient is still with no evidence of malignant disease.

\section{DISCUSSION}

Tonsillectomy specimens are routinely sent for histopathologic examination throughout the world as well as in the Czech Republic. Notably occult malignancy and risk of legal consequences argue in favor of this procedure. But recent literature shows relatively low incidence of incidental cancer in the tonsils. Many authors deal with this hot topic of cost abuse trying to strong argue against routine microscopic examination of resected tonsils. Ikram et al. described one malignant tumor in 400 tonsillar specimens: a non-Hodgkin's lymphoma in one tonsil of a patient who was a cigarette smoker and who had asymmetric tonsils [3]. The largest published study contains 2743 pediatric patients under the 


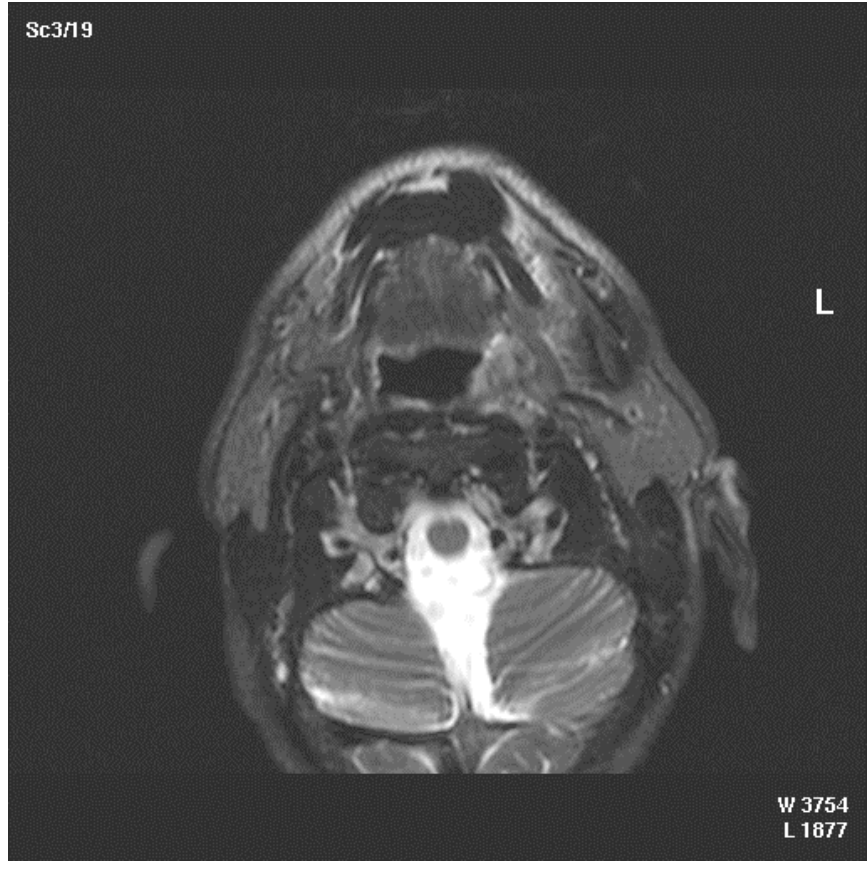

Fig. (1). Axial MRI section three weeks after UPPP shows asymmetry of the pharynx.

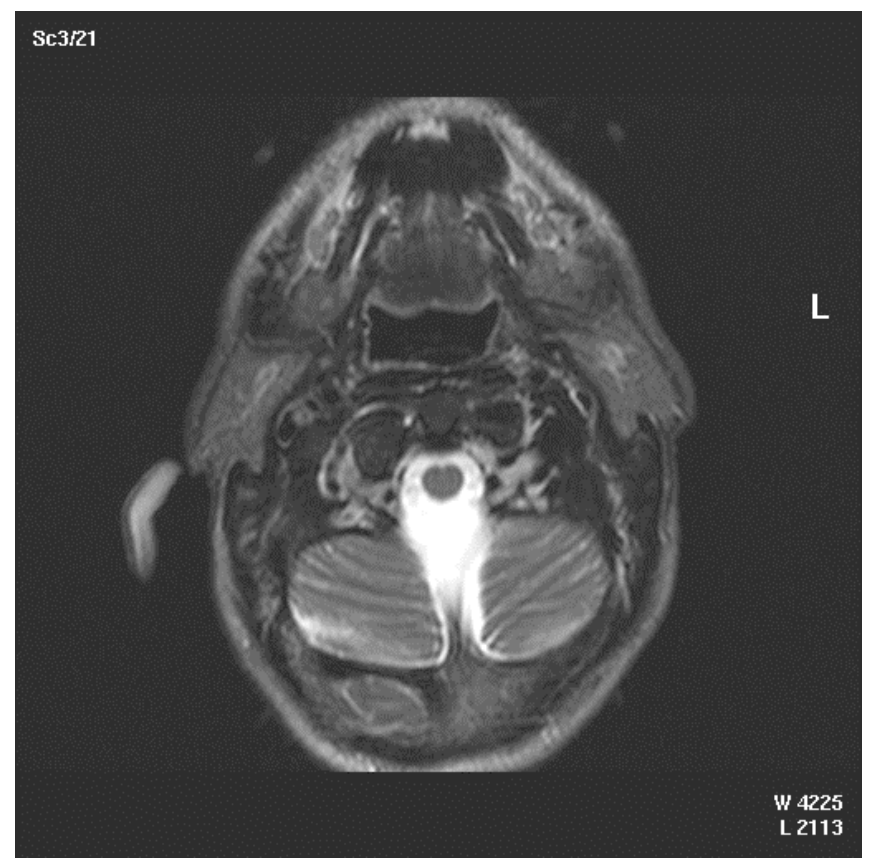

Fig. (2). Axial MRI section three months after surgery reveals no infiltration of the pharynx.

age of 19 operated for chronic or recurrent infections and obstructive hypertrophy. Evaluation of the pathology reports revealed no malignancies in tonsils [4]. Two cases of nonHodgkin's lymphoma were detected in Italian study of 1123 patients aged less than 16 years who underwent routine tonsillectomy for non-malignant indication [5]. Beaty et al. found a higher rate for adult population: 25 malignancies in 476 samples (5.25\%) [6]. The largest adult series comes from the USA: In 1280 tonsillectomies performed for benign disease there were no malignancies but in 31 cases of tonsillar asymmetry, two cases with malignant lymphoma (6.5\%) were identified [7]. Alvi and Vartanian found one occult malignancy $(2.3 \%)$ in 43 patients older than 20 years [8]. However, positive samples had been acquired from patients with cancer risk factors. Syms et al. explored a group of 49 patients who underwent tonsillectomy with normal mucosa, no lymphadenopathy, and asymmetry of the tonsils. Two (4.8\%) of the 49 patients with unilateral enlargement had the presence of malignancy - B-cell lymphoma [9]. The higest rate of positive histopathology was published by Younis et al.: None of the children from 2099 had an unusual histopathology finding other than lymphoid hyperplasia. On the other hand, of the 339 adults, 34 had squamous cell carcinoma (10.3\%) and 6 had lymphoma (1.77\%); however, these findings were suspected preoperatively by history and clinical manifestations [10]. There is no published study on cancer incidence in resected tissue in sleep apnea patients. In our group of 142 tonsillar specimens obtained in last five years only by sleep apnea surgery there was 1 malignant case $(0.7 \%)$.

We may summarize that the risk ratio for children is much lower than in adults. Typical pediatric occult malignancy of the tonsils is lymphoma. At the age above 18 years positive histopathology shifts to squamous cell carcinoma and malignancy occurrence growths. Cancer incidence summarized from above mentioned papers in tonsillectomy specimens for normal clinical tonsillar findings is bellow $0.1 \%$, but physicians should still pay attention to the possibilities of the presence of malignancy in seemingly normal tonsils. On the other hand, in presence of preoperative risk factors (a visible tonsillar lesion and/or asymmetry, a neck mass, a history of cancer, a history of tobacco or/and alcohol abuse) risk of incidental cancer finding growths. Our previous as well as often world-wide institution's policy requires the routine histopathologic evaluation of each surgically removed tissue, especially in adults. A risk ratio in non-suspicious cases is still not zero. And both psychological and social-economical burden of late recognized malignancy of the patient within health service is substantial.

But a presented case of occult malignancy of normal size tonsil in the sleep apnea patient without any risk factors does not support persistance on our previous practice. The patient did not required any adjuvant surgical or oncological intervence. The information of malignant histopathology and subsequent examination procedures brought him significant physical and psychological burden. Randall et al. published in 2007 a recent and ultimate retrospective PubMed review regarding tonsillectomy and realted surgical pathology [11]. It includes 54,901 patients reported in 20 studies. They found 54 malignancies $(0.087 \%$ prevalence $)$. Of these, 48 ( $88 \%$ of the patients) had suspicious features such as tonsillar asymmetry, cervical lymphadenopathy, or abnormal tonsil appearance, preoperatively. The remaining six patients without any suspicious features (better representing true occult malignancy) were $0.011 \%$ of the total cases. Given the very low incidence of real occult malignancy in the absence of acknowledged risk factors, routine histologic evaluation of adenotonsillectomy specimens is not recommended.

We hypothesize that the postoperative asymmetry of the pharynx could have been caused by inflammatory postoperative healing, although without any patient's 
complaints. Fortunately no recurrence or regional spread of cancer developed so that the patient is still with no evidence of disease and without need of any further treatment.

A recommendation from our experience and the current literature review could sound: In the absence of preoperative risk factors (a visible tonsillar lesion and/or asymmetry, a neck mass, a history of cancer, a history of tobacco or/and alcohol abuse) routine histopathologic examination of tonsillectomy specimens is not necessary. It could increase healthcare costs and results in consumption of resources and time of both surgeon and pathologist.

\section{ACKNOWLEDGEMENTS}

We thank Jiří Lisý, M.D. from Department of Radiological Techniques, $2^{\text {nd }}$ Faculty of Medicine, Charles University, Faculty Hospital Motol, Prague, Czech Republic for providing us with imaging documentation. This work was supported by the Internal Grant Agency of MZCR NR 9474-3.

\section{REFERENCES}

Hörmann K, Erhardt T, Hirth K, Maurer JT. Modified uvula flap in therapy of sleep-related breathing disorder. HNO 2001; 49: 361-6.
[2] Sher AE, Schechtman KB, Piccirillo JF. The efficacy of surgical modifications of the upper airway in adults with obstructive sleep apnea syndrome. Sleep 1996; 19: 156-77.

[3] Ikram M, Khan MA, Ahmed M, Siddiqui T, Mian MY. The histopathology of routine tonsillectomy specimens: results of a study and review of literature. Ear Nose Throat J 2000; 79: 880-2.

[4] Erdag TK, Ecevit MC, Guneri EA, Dogan E, Ikiz AO, Sutay S. Pathologic evaluation of routine tonsillectomy and adenoidectomy specimens in the pediatric population: is it really necessary? Int $\mathrm{J}$ Pediatr Otorhinolaryngol 2005; 69: 1321-5.

[5] Garavello W, Romagnoli M, Sordo L, Spreafico R, Gaini RM Incidence of unexpected malignancies in routine tonsillectomy specimens in children. Laryngoscope 2004; 114: 1103-5.

[6] Beaty MM, Funk GF, Karnell LH, et al., Risk factors for malignancy in adult tonsils. Head Neck 1998; 20: 399-403.

[7] Reiter ER, Randolph GW, Pilch BZ. Microscopic detection of occult malignancy in the adult tonsil. Otolaryngol Head Neck Surg 1999; 120: 190-4.

[8] Alvi A, Vartanian AJ. Microscopic examination of routine tonsillectomy specimens: is it necessary? Otolaryngol Head Neck Surg 1998; 119: 361-363.

[9] Syms MJ, Birkmire-Peters DP, Holtel MR. Incidence of carcinoma in incidental tonsil asymmetry. Laryngoscope 2000; 110: 1807-10.

[10] Younis RT, Hesse SV, Anand VK. Evaluation of the utility and cost-effectiveness of obtaining histopathologic diagnosis on all routine tonsillectomy specimens. Laryngoscope 2001; 111: 2166-9.

[11] Randall DA, Martin PJ, Thompson LD. Routine histologic examination is unnecessary for tonsillectomy or adenoidectomy. Laryngoscope 2007; 117: 1600-4. 